\title{
First Accreditation of a Medical Informatics Program by the European Federation of Medical Informatics: Experiences
}

\author{
Elske AMMENWERTH ${ }^{\mathrm{a}, 1}$ \\ ${ }^{a}$ UMIT - Private University for Health Scienes, Medical Informatics and Technology, \\ Hall in Tirol, Austria
}

\begin{abstract}
Background: European universities offer a variety of programs in Medical Informatics. The Europen Federation of Medical Informatics (EFMI) offers accreditation of these programs. Objectives: To describe the process of EFMI accreditation of a new Austrian master's program and reflect on accreditation benefits. Methods: Reflection on feedback and experiences at UMIT TIROL Results: Accreditation needs quite some preparation but offers essential opportunities for self-reflection and feedback by international experts. Conclusion: Besides national accreditation, medical informatics programs can benefit from the accreditation through international organizations.
\end{abstract}

Keywords. Medical informatics, accreditation, quality management

\section{Introduction}

Modern health care is not thinkable without medical informatics, and consequently, job prospects for graduates in medical informatics are excellent. The number of universities that offer master's programs in medical informatics is increasing, with $>1.000$ programs in biomedical and health informatics and related specializations in Europe alone (http://efmi-ac2.bmhi-edu.org).

Accreditation determines whether a program meets specific minimum standards. National accreditation may not always include a useful international perspective on medical informatics education [1]. International accreditation may provide this international perspective and increase the attractiveness of programs for students.

To ensure transparency and quality of medical informatics programs in Europe, the European Federation of Medical Informatics (EFMI) launched an accreditation program of medical informatics programs in 2020 [2]. While the accreditation fees are comparably low, some effort still needs to be invested in such a formal accreditation process. European universities now have to consider whether this EFMI accreditation is worthwhile for them.

This contribution aims to present the process and experiences of the first EFMI accreditation of a European medical informatics master's program and discuss benefits for European universities.

\footnotetext{
${ }^{1}$ Corresponding Author: Elske Ammenwerth, Institute of Medical Informatics, UMIT TIROL, Hall in Tirol, Austria, elske.ammenwerth@umit.at
} 


\section{Methods}

The first EFMI accreditation was conducted in summer 2020 for the new master's program Medical Informatics (http://www.umit.at/medical-informatics) at UMIT TIROL. The accreditation process comprised the following steps: Submission of the application to the EFMI board; appointment of an EFMI accreditation panel consisting of three members; preparation of a self-assessment report; panel visits in the educational institution (due to the pandemic situation, this was done online); preparation of an onsite panel's report: and decision on accreditation. Accreditation criteria comprised needs and relevance of the program, intended learning outcomes, academic environment, organization and implementation, and internal quality assurance and development. Overall, the accreditation process lasted less than four months. The self-assessment report comprised nearly 80 pages and was complemented by 22 Annexes. During the onsite visit, ten staff members from UMIT TIROL were interviewed. In the end, full EFMI accreditation was granted for the maximum possible duration of three years.

\section{Results}

The self-assessment report's preparation was more time-intensive than initially expected. Several aspects such as the institution, the new program's need, the intended learning outcomes, the teaching environment, organization, and quality assurance had to be described in detail and in English. However, the self-assessment report helped us reflect on our strengths, which helped us prepare national accreditation. The on-site discussions elaborated on several essential aspects of the program, and the experts' feedback helped us optimize curriculum and organization. For example, the feedback showed that the curriculum did not sufficiently show how it implemented some intended learning outcomes. It was helpful that our curriculum was in line with international education recommendations such as the IMIA recommendation [3].

\section{Discussion}

We benefited from the feedback of international experts on the content and organization of our curriculum. EFMI has a high reputation in medical informatics, and we expect that EFMI accreditation will help us attract the best national students. As UMIT TIROL was not involved in developing the EFMI accreditation procedure or criteria, this was also for EFMI the first "real life test" of their accreditation process.

\section{References}

[1] A. Hasman, and J. Mantas, IMIA accreditation of health informatics programs, Healthc. Inform. Res. 19 (2013) 154-161. doi:10.4258/hir.2013.19.3.154.

[2] J. Mantas, The Accreditation Procedure Ensures Quality of Education in Biomedical and Health Informatics., Stud. Health Technol. Inform. 272 (2020) 484-486. doi:10.3233/SHTI200601.

[3] J. Mantas, E. Ammenwerth, G. Demiris, A. Hasman, R. Haux, W. Hersh, E. Hovenga, K.C. Lun, H. Marin, F. Martin-Sanchez, and G. Wright, Recommendations of the international medical informatics association (IMIA) on education in biomedical and health informatics, Methods Inf. Med. 49 (2010). 